Ferre-Rey, G.; Dueñas, J.M.; Camps, C. (2021). Differences between the Dynamic and Normative Psychomotricity in Child Development. Revista Internacional de Medicina y Ciencias de la Actividad Física y el Deporte vol. 21 (81) pp. 47-62 Http://cdeporte.rediris.es/revista/revista81/artdiferencias1218.htm

DOI: https://doi.org/10.15366/rimcafd2021.81.004

\title{
ORIGINAL
}

\section{DIFERENCIAS ENTRE LA PSICOMOTRICIDAD DINÁMICA Y NORMATIVA EN EL DESARROLLO INFANTIL}

\section{DIFFERENCES BETWEEN THE DYNAMIC AND NORMATIVE PSYCHOMOTRICITY IN CHILD DEVELOPMENT}

\author{
Ferre-Rey, G. ${ }^{1}$; Dueñas, J.M. ${ }^{2}$ y Camps, C. $^{3}$ \\ ${ }^{1}$ Investigadora predoctoral, Universitat Rovira i Virgili (España) gisela.ferre@urv.cat \\ 2 Investigador de acceso al SECTI, (Sistema Español de Ciencia, Tecnología e Innovación). \\ Universitat Rovira i Virgili (España) jorgemanuel.duenas@urv.cat \\ ${ }^{3}$ Profesora titular de universidad, Universitat Rovira i Virgili (España) cori.camps@urv.cat
}

Código UNESCO / UNESCO code: 610000 Psicología / Psychology Clasificación Consejo de Europa / Council of Europe classification: 4. Educación Física y deporte comparado / Physical Education and sport compared

Recibido 18 de febrero de 2019 Received February 18, 2019

Aceptado 7 de julio de 2019 Accepted July 7, 2019

\section{RESUMEN}

La psicomotricidad en los contextos escolares puede ser aplicada según dos corrientes: la psicomotricidad normativa y la psicomotricidad dinámica. Aunque hay investigaciones de los beneficios de cada una de estas corrientes de psicomotricidad por separado, no hay estudios que hagan una comparativa entre ambas. El objetivo de la presente investigación fue establecer diferencias significativas entre ambas corrientes de psicomotricidad en cuanto al nivel de desarrollo general y psicomotriz en niños en edad preescolar. A la vez, se pretendió ver si había diferencias emocionales entre los niños de 5 años. Se evaluó mediante test estandarizados a 75 niños de educación infantil (entre 3 y 5 años) de dos escuelas públicas, donde el $50,7 \%$ de la muestra fueron niñas. Los resultados indicaron que, en general, había diferencias significativas a favor del grupo de psicomotricidad dinámica que de la psicomotricidad normativa. 
PALABRAS CLAVE: Psicomotricidad dinámica, psicomotricidad normativa, psicomotricidad educativa, desarrollo general, nivel psicomotriz, nivel emocional

\section{ABSTRACT}

Psychomotricity in school contexts can be applied according to two currents: normative psychomotricity and dynamic psychomotricity. Although there has been research into the benefits of each one of these psychomotricity currents separately, there are no studies that compares the two types with one another . The aim of the present study was to establish if there were any significant differences between the two currents of psychomotricity at the level of general and psychomotor development in children at preschool age. At the same time, the study aimed to determine any emotional differences among 5-year-old children. Seventy-five pre-school children (aged between 3 and 5 years) were evaluated through standardised tests in two public schools, where $50.7 \%$ of the sample were girls. The results indicated that, in general, psychomotor development was significantly better in the group that had followed the dynamic methodology than in the group that had been taught using the normative methodology.

KEY WORDS: Dynamic psychomotricity, normative psychomotricity, educational psychomotricity, general development, psychomotor level, emotional level

\section{INTRODUCCIÓN}

Según el Fórum Europeo de Psicomotricidad y la Federación de Asociaciones de Psicomotricistas del Estado Español, la psicomotricidad es una disciplina que atiende a la interacción que se establece entre cognición, emoción, cuerpo y movimiento, y que incide en el desarrollo global de la persona y en su capacidad para la expresión y relación en su contexto social. La psicomotricidad parte de una visión global del ser, y puede ser llevada a cabo en diferentes campos de actuación debido a que se ajusta al momento evolutivo, de desarrollo y competencial de las personas, independientemente de la edad. Por esta razón, existen diferentes niveles de intervención. En la actualidad, los campos de actuación de la psicomotricidad se centran en la educación, rehabilitación, terapia, prevención, salud y servicios sociales, los cuales van dirigidos a personas con diferentes tipologías: personas con un desarrollo evolutivo normalizado, personas con discapacidad, trastornos u otras dificultades tanto físicas, cognitivas, conductuales, del aprendizaje, del desarrollo y de la adaptación, y personas en situaciones de vulnerabilidad (European Forum of Psychomotricity, 2014; Mendiara y Gil, 2003; Mila, 2005).

En la actualidad, la psicomotricidad educativa suele ser más relevante y tener mejor acogida en la etapa que comprende de los 0 a los 6 años (etapas preescolar-infantil) (Rivas y Madrona, 2016). La psicomotricidad en contextos educativos tiene como objetivo facilitar el desarrollo y la maduración en la 
infancia, dentro del currículum escolar por medio del juego y la motricidad (Mendiara y Gil, 2003). Una prioridad de la psicomotricidad es el trabajo de relación de la propia persona con ella misma, con los objetos y con las demás personas (Berruezo, 2000). Existen diferentes vertientes teóricas que explican la intervención práctica de la psicomotricidad educativa. Ballesteros (1982) propuso dos corrientes: a) psicopedagógica o normativa representada por autores como J. Le Boulch o P. Vayer y b) vivencial o dinámica encabezada por A. Lapierre o B. Aucouturier. Esta diferenciación entre psicomotricidad normativa y dinámica es nombrada de otras formas por otros autores (por ejemplo, psicomotricdad instrumental y psicomotricidad relacional, según Serrabona, 2016), pero las características que definen a cada una de las corrientes son en esencia las mismas.

La corriente de psicomotricidad normativa parte de la idea que las sesiones deben ser dirigidas, donde los aprendizajes de los conceptos 0 de determinados parámetros psicomotores (como esquema corporal, orientación espacio-temporal, etc), se adquieran mediante ejercicios, juegos o actividades que han estado planificados y organizados con anterioridad. La sesión se estructura en función de los contenidos a trabajar, dedicando un tiempo a cada uno de ellos. La sesión es dirigida y todos los niños hacen las mismas actividades. Por otro lado, la corriente de psicomotricidad dinámica, parte de la idea que la sesión no debe estar dirigida, debe darse desde la acción y el juego espontáneo del niño, con el fin de favorecer la expresividad psicomotriz. De esta manera, no hay actividades programadas fijas, pero sí una planificación sobre la distribución de espacios, materiales y propuestas ajustados al grupo de niños y su relación con los contenidos curriculares. Así, la psicomotricidad dinámica da importancia al contexto de la práctica, como también a la distribución de los espacios y de los tiempos o fases de la sesión, y especialmente a las actitudes y estrategias del psicomotricista. También tiene en cuenta la acción y el origen del deseo del niño (Del Arco, 2017). Por ello, en este último enfoque se da especial énfasis a la formación personal y corporal del psicomotricista (Camps, 2008; Camps, Mila, García, Pecelli, Tomás y Bottini, 2011; García y Camps, 2006; Lapierre, Llorca, y Sánchez, 2015; Mila, 2008; Sánchez y Llorca, 2008a).

Silva, Neves y Moreira (2016) llevaron a cabo una investigación a través de sesiones de psicomotricidad normativa y encontraron que éstas favorecían un mayor desarrollo de habilidades motoras y mayor motivación de los y las preescolares con respecto a las actividades académicas. Resultados parecidos encontraron Gutiérrez, Fontenla y Cons (2017) con respecto a una mejora de la inteligencia emocional y autoestima mediante este tipo de psicomotricidad.

Por otro lado, Cró, Andreucci, Pereira y Pinho (2011) aplicaron programas estructurados, dentro de los cuales había uno de psicomotricidad normativa con un grupo de niños preescolares de familias con un poder adquisitivo bajo y con baja estimulación. Vieron que los niños que participaron en estos programas tuvieron mejores puntuaciones en las habilidades personales y sociales, y mejoraron su calidad de vida, su bienestar y rendimiento académico. Moreira, Almeida y Marinho (2016) también aplicaron en su estudio un programa de psicomotricidad educativa estructurada, donde vieron que 
mejoraba la auto-percepción de la competencia física en los niños, y que había una repercusión positiva en las actividades académicas. También confirman la mejora de habilidades a través de la psicomotricidad normativa Kambas et al. (2010). En este caso, con niños de entre 4 y 6 años, constataron que un programa de psicomotricidad normativa repercutió en una mejor habilidad en el control viso-motor que aquellos niños que no participaron.

Todas estas investigaciones muestran que la aplicación de la psicomotricidad educativa normativa repercute en una mejora del rendimiento de diferentes aspectos en los niños, en concreto en la población preescolar, objeto del presente estudio. Pero en ellos no se realiza una comparación entre ambos tipos de metodología psicomotriz, normativa y dinámica, sino que se realizan sesiones de educación psicomotriz de tipo normativo únicamente y se analizan los efectos de las mismas.

No obstante, hay pocos estudios que se hayan realizado con una práctica psicomotriz educativa de corriente dinámica. Por ejemplo, Mas y Castellà (2016) analizaron los efectos de sesiones de práctica psicomotriz dinámica en niños de entre 11 y 22 meses que realizaban dos sesiones semanales. Los resultados revelaron que estos niños obtenían mejores puntuaciones en el desarrollo general, cognición y habilidades motoras en comparación con otro grupo de niños que solo realizaban una sesión semanal. A su vez, compararon los resultados con niños que no realizaban ninguna sesión, obteniendo siempre mejores resultados el grupo con mayor frecuencia de sesiones, por lo que se sugiere que la práctica psicomotriz dinámica tiene implicaciones en el óptimo desarrollo evolutivo y que estos beneficios se incrementan al aumentar las sesiones. También se han hecho propuestas teóricas sobre la realización de sesiones de práctica psicomotriz educativa desde la corriente dinámica en edad preescolar, como la Del Arco (2017) y de estrategias y recursos que pueden utilizar los y las profesionales de la psicomotridad dinámica en este campo educativo (Arnaiz, Rabadán y Vives, 2001; Aucouturier, 2004, 2018; Rota, 2015; Sánchez y Llorca, 2008b).

Analizando los resultados de las diferentes investigaciones que hemos citado, podemos afirmar que la realización de psicomotricidad educativa, independientemente si es de corriente normativa o dinámica, aporta una serie de beneficios en el desarrollo de los niños en edad preescolar, en habilidades motoras, personales, sociales, cognición, inteligencia emocional, motivación y rendimiento en las tareas académicas, desarrollo general, autopercepción de la competencia física, autoestima, bienestar, control viso-motor y mejora de la calidad de vida. Según Lupu (2011) la educación psicomotriz es la base para un desarrollo físico y psicológico armonioso, y por ello se debe implementar desde educación infantil. Además, como apuntan Solís, Prieto, Nistal y Vázquez (2017) en su estudio, la mayor parte de profesionales que imparten psicomotricidad educativa consideran que esta disciplina es fundamental para trabajar el desarrollo global infantil y que debe haber una planificación de las sesiones previamente. Para Mamani (2016), cuando se aplica de manera apropiada la técnica psicomotriz facilita que los niños mejoren sus habilidades en clase, y, además, la utilización de materiales adecuados posibilita que se desarrollen las competencias motrices. Recalcan asimismo la importancia de 
una planificación previa que tenga en cuenta la situación social y el nivel de desarrollo de cada niño.

Sin embargo, no hay estudios que comparen estas dos corrientes de psicomotricidad y si una obtiene mejores resultados en el desarrollo de los niños que la otra. Terry (2014) planteó una comparativa entre psicomotricidad normativa y dinámica en niños de entre 3 y 4 años en aptitudes motrices mediante las tres subescalas de la Batería McCarthy (McCarthy, 1977): coordinación de piernas, coordinación de brazos y acción imitativa, y comprobó que los niños obtuvieron mayores puntuaciones en aptitudes motrices con la realización de psicomotricidad normativa que con la psicomotricidad dinámica. No obstante, el procedimiento utilizado muestra que la psicomotricidad dinámica no fue llevada a cabo por una persona experta en este tipo de práctica psicomotriz, por lo que los resultados del estudio pueden estar sesgados.

Por ello, se plantearon los siguientes objetivos para la presente investigación: establecer si hay diferencias significativas entre la práctica de la psicomotricidad dinámica y la psicomotricidad normativa en el desarrollo general y psicomotriz de niños de edades preescolares (de 3 a 5 años) y, en caso de haberlas, cuál es la metodología más óptima. Además, se plantea analizar las diferencias emocionales en niños de 5 años.

\section{MATERIAL Y MÉTODO}

\section{PARTICIPANTES}

La muestra está formada por 75 niños de educación infantil, con edades comprendidas entre los 3 y 5 años, de dos escuelas públicas de la provincia de Tarragona (España). Específicamente, el 50,7 \% de la muestra fueron niñas. El $28 \%$ fueron alumnos de Parvulario 3, el 42,7\% alumnos de Parvulario 4 y el $29,3 \%$ alumnos de Parvulario 5. Ambas escuelas acogen a familias con un nivel socioeconómico medio-bajo, sin llegar a ser consideradas escuelas de alta complejidad. El tipo de psicomotricidad (normativa o dinámica) fue clasificada en relación a la formación de la persona que impartía las sesiones de psicomotricidad, su experiencia y el protocolo de las sesiones. Las dos personas formadoras tenían formación de postgrado en su respectiva corriente y los años de experiencia fueron similares. Además, el equipo de investigación comprobó una de las sesiones previas, con la finalidad de definir el tipo de práctica psicomotriz.

\section{MEDIDAS}

Con la finalidad de cumplir los objetivos propuestos se administró una batería de cuestionarios que se describen a continuación:

Inventario de Desarrollo Battelle (Newborg, Stock y Wnek, 1998): este inventario evalúa el nivel de desarrollo en la infancia desde los 0 a los 95 meses de edad. Se evalúan cinco áreas: personal/social (capacidad para 
realizar interacciones sociales significativas), adaptativa (mide autonomía en áreas como en el vestir, alimentación, aseo, y capacidad para responsabilizarse), motora (uso y control corporal, se divide en área motora gruesa y motora fina), comunicación (lenguaje expresivo y lenguaje comprensivo) y cognitiva (evalúa la capacidad conceptual). Este test se puede aplicar de forma completa o con la forma abreviada (cribado). En este estudio se utilizó la forma de cribado, ya que nos da información general del nivel de desarrollo del niño con un menor tiempo. Para cada puntuación directa de las diferentes áreas y la puntuación total se muestran sus edades equivalentes.

Test Psicomotor de Picq y Vayer (Picq y Vayer, 1977): es una prueba que evalúa el nivel psicomotor en la infancia entre los 2 y los 11 años de edad. En nuestro caso, hemos administrado la parte del examen psicomotor de la primera infancia, ya que evalúa cada conducta entre los 2 y los 5 años, siendo más ajustada a la edad de nuestra muestra. Las conductas que evalúa son la coordinación visomanual (organización de movimientos en los cuales la visión y los miembros superiores trabajan a la vez), la coordinación dinámica (organización de movimientos en los cuales están implicados diversos grupos musculares a la vez y tienen una finalidad), el control postural (equilibrio), el control del propio cuerpo (esquema corporal y praxias), la organización perceptiva (interpretación y estructuración de la información proveniente de nuestros sentidos), y el lenguaje (memoria inmediata y pronunciación), y se complementa con una prueba de lateralidad (tendencia a utilizar un lado del cuerpo con preferencia a la otra).

Test del dibujo de la figura humana (Koppitz, 1984): mediante la realización del dibujo de una persona se evalúan una serie de ítems evolutivos (signos que están en relación con la edad y el nivel madurativo) y unos ítems que representan indicadores emocionales (signos relacionados con las actitudes y preocupaciones de los niños), la evaluación se realiza en función de la calidad de los dibujos, los detalles especiales que no se suelen dibujar, y las omisiones de ítems esperables a una determinada edad. En esta investigación se utilizó el test del dibujo de la figura humana con el fin de explorar el nivel emocional de los niños de parvulario 5. Esto se debió a que los baremos de este test empiezan a los 5 años.

\section{PROCEDIMIENTO}

El presente estudio ha tenido en consideración las recomendaciones de la ley orgánica española 15/1999 y la Agencia Española de Protección de Datos, que regulan el derecho fundamental a la protección de datos. Por lo que se siguieron diferentes directrices que se exponen a continuación. En primer lugar, el equipo de investigación definió los criterios de inclusión y exclusión de las escuelas participantes. Se estableció que el personal docente debía tener formación de postgrado específica en el tipo de psicomotricidad que realizaba (mínimo de 30 créditos), además, experiencia de mínimo dos años.

Después se contactó con seis escuelas públicas al azar por medio de muestreo probabilístico simple. El personal directivo de cinco escuelas accedió a entrevistarse con el equipo de investigación. En la reunión, se explicaron los 
objetivos del estudio y las implicaciones para la escuela, además, se les preguntó el tipo de intervención psicomotriz que realizaban, formación de la persona docente, años de experiencia y procedimiento utilizado.

Posteriormente, se reunió el equipo de investigación y evaluaron los criterios de inclusión. Solo una escuela utilizaba intervención psicomotriz dinámica y reunía los criterios. De las cuatro escuelas restantes dos no cumplían los criterios. Con la finalidad de equiparar la muestra se eligió a la escuela que tenía características sociodemográficas similares.

Una vez seleccionadas las escuelas, se enviaron consentimientos informados a las familias de todos los niños, junto con información sobre los objetivos e implicaciones del estudio. Solamente fueron evaluados los niños que sus familias dieron el consentimiento. Aun así, el niño era libre de no querer participar o declinar el consentimiento en cualquier momento. Las pruebas fueron aplicadas por profesionales graduados en Psicología y con posesión de un título de Máster en Psicomotricidad y con experiencia en la aplicación de este tipo de pruebas, que integraban el equipo de esta investigación.

En cuanto a los análisis estadísticos, la normalidad de los datos se analizó mediante la prueba de Kolmogorov-Smirnov $(p>0.05)$ por lo que se decidió realizar pruebas paramétricas. Posteriormente, se llevó a cabo una comparación de medias a través del procedimiento $t$ de Student para dos muestras independientes, con la finalidad de identificar las diferencias que la práctica psicomotriz podría tener en el desarrollo socioemocional de los niños.

\section{RESULTADOS}

En la Tabla 1 se muestran los estadísticos descriptivos de las diferentes áreas y subáreas de la prueba de cribado del inventario de desarrollo Battelle y la prueba $t$ para dos muestras independientes. Los resultados muestran que el grupo de psicomotricidad dinámica tiene mayores medias en la mayoría de las áreas evaluadas a través del inventario de desarrollo de Battelle en comparación con el grupo de psicomotricidad normativa. Únicamente las áreas Personal-Social y Adaptativa no presentan diferencias significativas entre los grupos. Por otra parte, como se puede observar, los valores de asimetría y curtosis indican una adecuada distribución de los datos para realizar análisis paramétricos. 
Tabla 1. Estadísticos descriptivos en el inventario de desarrollo Battelle

\begin{tabular}{|c|c|c|c|c|c|c|c|c|c|c|}
\hline \multirow[b]{2}{*}{ Variables } & \multirow[b]{2}{*}{ Media } & \multicolumn{3}{|c|}{ Muestra general } & \multicolumn{2}{|c|}{$\begin{array}{l}\text { Psicomotricidad } \\
\text { Normativa }\end{array}$} & \multicolumn{2}{|c|}{$\begin{array}{l}\text { Psicomotricidad } \\
\text { Dinámica }\end{array}$} & \multicolumn{2}{|c|}{$\begin{array}{l}\text { Prueba para la } \\
\text { igualdad de } \\
\text { medias }\end{array}$} \\
\hline & & $D . T$ & Asimetría & Curtosis & Media & $D . T$ & Media & $D . T$ & $t$ & $p$ \\
\hline \multicolumn{11}{|l|}{ Áreas } \\
\hline P.S & 33,6 & 4,6 & $-1,1$ & $-0,7$ & 34,81 & 4,1 & 33,15 & 4,8 & 2,3 & 0,13 \\
\hline$A$ & 29,7 & 3,7 & 0,2 & $-0,5$ & 29,41 & 3,1 & 29,92 & 4,1 & 0,3 & 0,58 \\
\hline$M$ & 13,6 & 5,5 & $-0,4$ & $0-, 4$ & 27,48 & 5,1 & 32,13 & 5 & 14,7 & $<.001$ \\
\hline $\mathrm{C}$ & 25,7 & 6,1 & $-0,2$ & $-0,9$ & 21,48 & 3,3 & 28,1 & 6,2 & 26,8 & $<.001$ \\
\hline $\mathrm{CO}$ & 25,6 & 5,8 & $-0,2$ & $-1,5$ & 22,67 & 6,3 & 27,29 & 4,9 & 12,6 & $<.001$ \\
\hline \multicolumn{11}{|l|}{ Subáreas } \\
\hline M.G & 13,6 & 2,6 & $-0,4$ & $-0,6$ & 12 & 2,3 & 14,58 & 2,4 & 21,3 & $<.001$ \\
\hline M.F & 16,8 & 3,4 & $-0,5$ & $-0,3$ & 15,48 & 3,3 & 17,54 & 3,4 & 6,6 & 0,01 \\
\hline C.R & 12,8 & 3,3 & $-0,6$ & $-1,2$ & 10,04 & 1,9 & 14,38 & 2,9 & 47,3 & $<.001$ \\
\hline C.E & 18,9 & 3,2 & $-0,6$ & 0,27 & 11,44 & 1,8 & 13,73 & 3,6 & 9,7 & $<.001$ \\
\hline D.G & 30,45 & 18,6 & $-0,4$ & $-0,1$ & 136,22 & 14,85 & 150,5 & 18,59 & 11,7 & $<.001$ \\
\hline
\end{tabular}

Nota. P.S=personal social, $\mathrm{A}=$ adaptativa, $\mathrm{M}=$ Motor, $\mathrm{C}=$ comunicación, $\mathrm{CO}=$ cognitiva, $\mathrm{MG}=$ motora gruesa, $\mathrm{MF}=$ motora fina, $\mathrm{CR}=$ comunicación receptiva, $\mathrm{C}$.E=comunicación expresiva, $\mathrm{DG}=$ desarrollo global

En la tabla 2 se presentan los estadísticos descriptivos para las diferentes áreas del test Picq y Vayer. Los resultados sugieren que el grupo de psicomotricidad dinámica obtiene puntuaciones significativamente mayores que las del grupo de psicomotricidad normativa, excepto en las escalas de coordinación visomanual y lateralidad, donde no se aprecian diferencias significativas.

Tabla 2. Estadísticos descriptivos en el test Picq y Vayer

\begin{tabular}{|c|c|c|c|c|c|c|}
\hline \multirow[b]{2}{*}{ Variables } & \multicolumn{2}{|c|}{$\begin{array}{l}\text { Psicomotricidad } \\
\text { normativa }\end{array}$} & \multicolumn{2}{|c|}{$\begin{array}{l}\text { Psicomotricidad } \\
\text { Dinámica }\end{array}$} & \multicolumn{2}{|c|}{$\begin{array}{l}\text { Prueba para la igualdad de } \\
\text { medias }\end{array}$} \\
\hline & Media & $D . T$ & Media & $D . T$ & $t$ & $p$ \\
\hline $\begin{array}{l}\text { Coordinación } \\
\text { visomanual }\end{array}$ & 4,13 & 1,05 & 4,49 & 1,27 & 1,56 & 0,22 \\
\hline $\begin{array}{l}\text { Coordinación } \\
\text { dinámica }\end{array}$ & 4,17 & 1,32 & 4,94 & 1,26 & 6,23 & 0,01 \\
\hline Control postural & 4,43 & 1,68 & 5,31 & 1,39 & 6,06 & 0,02 \\
\hline Control propio cuerpo & 12,63 & 3,02 & 16,73 & 1,85 & 53,3 & $<.001$ \\
\hline $\begin{array}{c}\text { Organización } \\
\text { perceptiva }\end{array}$ & 3,85 & 0,53 & 4,21 & 0,77 & 4,54 & 0,04 \\
\hline Lenguaje & 3,85 & 1,13 & 4,46 & 0,71 & 8,09 & 0,01 \\
\hline Lateralidad & 4,93 & 1,66 & 4,35 & 2,09 & 1,49 & 0,23 \\
\hline
\end{tabular}

En la tabla 3 se muestran las puntuaciones de los indicadores emocionales del test de la Figura Humana en los niños de parvulario 5. Los datos sugieren que no hay diferencias significativas en ninguna de las áreas. 
Tabla 3. Estadísticos descriptivos en test de la Figura Humana

\begin{tabular}{|c|c|c|c|c|c|c|}
\hline & \multicolumn{2}{|c|}{$\begin{array}{l}\text { Psicomotricidad } \\
\text { normativa }\end{array}$} & \multicolumn{2}{|c|}{$\begin{array}{l}\text { Psicomotricidad } \\
\text { dinámica }\end{array}$} & \multicolumn{2}{|c|}{$\begin{array}{l}\text { Prueba para la igualdad de } \\
\text { medias }\end{array}$} \\
\hline Variables estudiadas & Media & D.T & Media & $D . T$ & $t$ & $p$ \\
\hline Signos cualitativos & 1,75 & 1,39 & 1,57 & 0,94 & 0,13 & 0,72 \\
\hline Detalles especiales & 1,50 & 1,20 & 1,14 & 1,03 & 0,55 & 0,47 \\
\hline Omisiones & 0,50 & 1,07 & 0,29 & 0,47 & 0,43 & 0,52 \\
\hline $\begin{array}{l}\text { Indicadores } \\
\text { emocionales }\end{array}$ & 3,75 & 1,28 & 3,00 & 1,47 & 1,45 & 0,24 \\
\hline
\end{tabular}

\section{DISCUSIÓN}

En el presente estudio se planteó como objetivo establecer si había diferencias significativas entre la práctica de la psicomotricidad normativa y la psicomotricidad dinámica en el desarrollo general y psicomotriz en edad preescolar y, en caso de haberlas, cuál era el más óptimo. Además, analizar las diferencias emocionales en niños de 5 años.

Se ha comparado dos grupos de niños preescolares con dos metodologías de práctica psicomotriz diferente, un grupo con una metodología de tipo normativo dinámica y el otro de tipo dinámico, división que señaló Ballesteros (1982).

Teniendo en cuenta las diferencias de metodología de ambas prácticas psicomotrices (Del Arco, 2017), los resultados sugieren que los niños que estuvieron expuestos a sesiones de psicomotricidad dinámica obtuvieron mejores resultados en el desarrollo general y a nivel psicomotor que los niños que realizaron psicomotricidad normativa. Se han encontrado mejores resultados en el área motora, la subárea motora gruesa, la subárea motora fina, el área de comunicación, la subárea de comunicación receptiva, la subárea de comunicación expresiva, el área cognitiva, el desarrollo global, la coordinación dinámica, el control postural, el control del propio cuerpo, la organización perceptiva y en el lenguaje. Estas áreas están estrechamente vinculadas a la idea de globalidad del que parte el concepto de psicomotricidad y la consideración de la persona como una totalidad, en especial en la etapa de 0 a 6 años, en la cual los aspectos motrices, cognitivos, emocionales y sociales están estrechamente vinculados. Tal y como comenta Camps (2009), las distintas definiciones de psicomotricidad destacan la incidencia de la educación psicomotriz sobre la totalidad del ser, estando los aspectos psíquicos y motores profundamente interrelacionados. Así, la intervención psicomotriz, favorece un desarrollo equilibrado de la personalidad del niño y contribuye a su construcción psíquica.

Los resultados muestran puntuaciones más altas en los distintos ámbitos de desarrollo de los niños y también en relación al desarrollo global. En relación al desarrollo motor vemos puntuaciones más altas, concretamente en motricidad gruesa (Battelle), coordinación dinámica, control postural, y control del propio 
cuerpo (Picq y Vayer), es decir, a nivel de coordinación dinámica general y equilibrio, pero no en relación a la motricidad fina o visomotora. Más abajo nos referiremos a ello. En relación a la motricidad gruesa creemos que el hecho de que en estas sesiones los niños se desplazan, corren, saltan, etc, siempre a partir de su propio momento evolutivo y competencial puede favorecer una zona de desarrollo próximo (Chaiklin, 2003), no forzando aprendizajes motrices para los cuales todavía no está maduro o llevando a cabo actividades que tienen poca resonancia emocional porque ya las tienen superadas y no necesitan poner atención en ellas.

También se dan puntuaciones superiores en el área cognitiva (Battelle), en la organización perceptiva y en el lenguaje (Picq-Vayer). Aunque serían necesarias muchas más evidencias, podemos hipotetizar que la corriente dinámica, al trabajar globalmente todos los aspectos del desarrollo infantil, partiendo del propio juego y del propio deseo, favorece el desarrollo cognitivo a nivel de discriminación perceptiva, memoria, razonamiento, y desarrollo conceptual, que son las habilidades y capacidades implicadas en las pruebas aplicadas.

Las puntuaciones son también superiores en el área de comunicación (Battelle). Concretamente la subárea de comunicación receptiva, que evalúa la discriminación, reconocimientos y comprensión de sonidos y palabras, así como la información precisa a través de estos, signos, sistema Braille u otros medios no verbales; y la subárea de comunicación expresiva, que evalúa la producción y uso de sonidos, palabras y gestos como medio para transmitir información a los demás. Creemos que ello puede verse facilitado por el hecho de que en la corriente dinámica se da mucha importancia a la expresión espontánea de los niños y el psicomotricista utiliza de forma significativa los distintos mediadores de comunicación (mirada, gestos, contacto, tono de voz, postura...), propiciando una comunicación no verbal además de la comunicación verbal que se da en ambas metodologías.

Las diferencias encontradas pueden deberse a la concepción de la psicomotricidad dinámica y su metodología. Como expresa Aucouturier (2015), el niño requiere de una maduración tónico-emocional para adquirir los conocimientos escolares y la cultura, y que no todos los niños lo logran a la misma edad. Defiende que en el juego libre fluyen la tonicidad, emociones, gestualidad, representaciones conscientes e inconscientes y surge el placer de actuar. Mediante el juego puede simbolizar el miedo, representarse a sí mismo y afirmar su existencia. Se trata de un juego que parte del deseo del niño y en el que encuentra un adulto psicomotricista disponible corporal y emocionalmente. Para Polacco (2015), la importancia de esta metodología reside en el juego espontáneo y la interacción con un adulto con un sistema de actitudes, desarrollado a partir de una formación específica en la que trabaja muy bien en su propio cuerpo en la relación consigo mismo y con el de los demás. Para él, gracias al juego, el mundo externo puede ser puesto en relación con el mundo interno y viceversa. Esto abre el camino para un posible cambio a nivel profundo. Para Polacco, el aspecto particular de la práctica psicomotriz de Aucouturier (metodología que se ha seguido con el grupo de psicomotricidad dinámica en esta investigación), comparado con todas las 
demás actividades que se basan en el juego, se refiere a la importancia que se da a las acciones en el juego y al hecho de que el placer que las acompaña puede, por sí solo, mitigar algunas situaciones dolorosas, que el niño puede haber vivido hasta entonces, y puede implicar un cambio profundo. Pero, tal como afirma, para ello el niño debe sentirse seguro frente a un adulto que le acompaña en el juego. Refiere el juego como uno de los 7 circuitos subcorticales con funciones de supervivencia, reproducción y desarrollo psicológico.

El trabajo que realiza el psicomotricista dinámico a partir de la experimentación libre del niño de su cuerpo, mediante el juego, y sus actitudes de escucha, disponibilidad y contención, así como la utilización de estrategias ajustadas y de los mediadores de comunicación (Camps, 2012), puede ser la explicación de estas diferencias, ya que un trabajo a nivel tónico-emocional con los niños preescolares tiene una repercusión en las otras áreas del desarrollo, tal y como se han visto en los resultados de esta investigación. Según Camps (2009), es la intervención sobre la globalidad del ser, la relación tónico-emocional que se establece con el niño y las actitudes del profesional en psicomotricidad dinámica lo que favorece que su desarrollo se dé de manera armoniosa y que ayude a la estructuración de su psiquismo.

Las áreas del desarrollo en las que no se encontraron diferencias, PersonalSocial y Adaptativa, podría ser debido a que estas áreas se trabajan diariamente en las escuelas, formando parte del área de autonomía personal, tal y como establece el decreto 181/2008. De hecho, revisando los ítems que corresponden a la aplicación del inventario de desarrollo Battelle para estas edades, observamos que éstos ítems remiten al aprendizaje de hábitos que se trabajan en educación infantil, por ejemplo, abrocharse botones, vestirse, en el caso del área Adaptativa, o de aprendizaje de conductas sociales, por ejemplo, reconocer las diferencias entre hombre y mujer, o distinguir conductas aceptables y no aceptables, en el caso del área Personal-Social.

En cuanto a las áreas de coordinación visomanual y lateralidad, tampoco se encontraron diferencias entre metodologías. Esto también puede deberse a que las escuelas trabajan diariamente estas áreas, ya que son la base de los aprendizajes posteriores. Así, si nos detenemos en los ítems aplicados en las pruebas de Battelle y de Picq y Vayer para estas edades, vemos que los mismos corresponden a aprendizajes y hábitos que se trabajan de forma habitual en educación infantil, por ejemplo, hacer una construcción con cubos, enhebrar una aguja, hacer un nudo, cortar con tijeras, o copiar una figura. Además, en el caso de la lateralidad, se ha visto que a las edades de nuestra muestra (de 3 a 5 años) no suele estar definida. Tal y como indican Mayolas y Reverter (2015), la lateralidad tiende a hacerse homogénea en gran parte de la población entre los 11 y 15 años, produciéndose un cambio significativo de la lateralidad cruzada a la homogénea alrededor de los 8-10 años. Por otro lado, en relación a la lateralidad manual, se encuentra definida en algunos casos al final del primer año, pero en general hay una alternancia de periodos de ambidextrismo, dominancia derecha o izquierda, con desplazamiento hacia la derecha entre los 2 y 3 años y hacia la izquierda entre los 3 y 5 años, 
constatándose una oscilación de la lateralidad hasta los 5 años y a veces hasta los 7 años en algunos niños (Sassano, 2015).

En cuanto a los indicadores emocionales del test de la Figura Humana vemos que no hay diferencias entre ambas metodologías. De hecho, la prueba aplicada (figura humana de Koppitz) es aplicable a partir de los 5 años y por tanto sólo pudimos hacer este análisis con este grupo de edad. No obstante, un indicador indirecto de aspectos emocionales es el control postural y equilibrio, ya que, tal como afirman distintos autores (D'Mello, Dale y Graesser, 2012; Lelard, Stins y Mouras, 2019) existe una íntima relación entre el control postural $y$ las emociones de las personas.

Así, podemos comprobar cómo en la prueba de Picq y Vayer los ítems que pertenecen a la escala "control postural" son pruebas de equilibrio, tal como la misma prueba indica, y en ella los niños del grupo de psicomotricidad dinámica tienen puntuaciones significativamente superiores. Igualmente, analizando los ítems de motricidad gruesa del inventario de desarrollo de Battelle, comprobamos que todos ellos son pruebas de equilibrio y no de coordinación dinámica. En este sentido, remitimos a distintos autores (Berruezo, 2002; D'Mello, Dale y Graesser, 2012; Lelard, Stins y Mouras, 2019) que destacan la relación entre el equilibrio y desarrollo emocional, relación que no se dan cuando hablamos de motricidad gruesa refiriéndonos a actividades de coordinación dinámica. Este aspecto creemos que es primordial a la hora de interpretar los resultados de pruebas que implican ítems de motricidad gruesa, pero que éstos pueden ser de coordinación dinámica o pueden implicar también el equilibrio y es imprescindible determinar qué contenido psicomotor es el que se está evaluando para poder hacer una interpretación de los resultados que sea ajustada. Ya Wallon (1978, en Berruezo, 2002) destacó la interrelación entre la actividad laberintico-propioceptiva o postural, por un lado, y la vida emocional por el otro. Para él, la vida afectiva tiene un substrato orgánico que se establece sobre funciones tónico-laberínticas y posturales. Se ha comprobado la relación existente entre las alteraciones del equilibrio y los estados de ansiedad, debido a las relaciones entre la vida afectiva y el fondo tónico. El sistema vestibular influye en las funciones emocionales, dadas sus intrincadas conexiones con el sistema límbico. El equilibrio, además, la base de toda coordinación dinámica, es un aspecto de la educación del esquema corporal y está vinculado a los procesos de atención (Berruezo, 2002; Sassano, 2014).

Por tanto, considerando esta relación del control postural con la esfera emocional, se puede ver cómo los niños que realizan psicomotricidad dinámica tienen valores más elevados que los niños que siguen psicomotricidad normativa. Esto sugiere un efecto positivo y mayor sobre la esfera emocional de los niños de educación infantil que realizan una práctica psicomotriz dinámica que con respecto a los de una práctica psicomotriz normativa. Para futuros estudios se podría evaluar las diferencias emocionales entre grupos mediante una metodología cualitativa, donde una persona experta en la interpretación de dibujos haga su análisis de cada dibujo y se pueda hacer una comparativa. 
Así, nuestros resultados están en la línea de investigaciones de Mas y Castellà (2016), en que apoyaban que este tipo de psicomotricidad favorecía el desarrollo general, las habilidades motoras y la cognición. También estos resultados están en contra de los resultados de Terry (2014), en que mostraba una superioridad en aptitudes motrices con una psicomotricidad normativa sobre la dinámica, aunque ya discutimos anteriormente que estos resultados podían estar sesgados.

\section{LIMITACIONES}

El presente estudio tiene algunas limitaciones. Por un lado, el diseño metodológico contempló una evaluación postest, esto no ha permitido observar el nivel de desarrollo inicial de los participantes. Esto se debió a que la muestra objeto de estudio son niños entre tres a cinco años y la exposición a dos evaluaciones en el mismo año consideramos excesivo. Sin embargo, se excluyeron de la muestra aquellos niños que presentaban trastornos del desarrollo o indicios de retraso en el desarrollo, por lo que se asume que todos los participantes tenían un desarrollo evolutivo normalizado. Además, tal y como apunta Terry (2014), en las primeras etapas de escolarización es frecuente que haya una homogenización de las habilidades motrices.

Por otro lado, aunque se valoró diferentes variables de los profesionales encargados de realizar las sesiones de psicomotricidad, como el nivel de formación y de experiencia, no se contemplaron factores personales que pudieran estar influyendo en los resultados, por ejemplo, rasgos de personalidad, contacto con el alumnado a parte de las sesiones de psicomotricidad y contacto cercano con las familias. Se necesitan estudios que evalúen y triangulen las características del psicomotricista con los beneficios en el desarrollo de los y las preescolares.

Del mismo modo, en el presente estudio se tuvo en cuenta las características socioeconómicas grupales de los estudiantes. Sin embargo, no se evalúo la situación socioeconómica individual de cada niño. Estos datos podrían dar luz a la literatura sobre la influencia de los diferentes tipos de psicomotricidad en situaciones sociales, económicas y culturales concretas.

\section{CONCLUSIONES}

La práctica de la psicomotricidad educativa se ha incrementado en los últimos años en las escuelas de educación infantil. Sin embargo, existen pocos estudios que analicen sus beneficios desde un modelo cuantitativo. Los datos de este estudio sugieren que la práctica psicomotriz dinámica proporciona unos beneficios a nivel psicomotriz y del desarrollo evolutivo adicionales a la práctica psicomotriz normativa, y posiblemente también a nivel emocional. Hacen falta más estudios que permitan comprobar los beneficios adicionales de la psicomotricidad dinámica y normativa de manera empírica.

\section{CONFLICTO DE INTERESES}

Los autores de este artículo declaran que no tienen ningún conflicto de intereses. 


\section{REFERENCIAS BIBLIOGRÁFICAS}

Arnaiz, P., Rabadán, M., y Vives, I. (2001). La psicomotricidad en la escuela: una práctica preventiva y educativa. Málaga: Ed. Aljibe.

Aucouturier, B. (2004). Los fantasmas de acción y la práctica psicomotriz. Barcelona: Ed. Graó.

Aucouturier, B. (2015). La práctica psicomotriz a nivel educativo, preventivo y terapéutico. Revista Latinoamericana de Educación Infantil, 4(2), 205211.

Aucouturier, B. (2018). Actuar, jugar, pensar. Barcelona: Graó.

Ballesteros, S. (1982). El esquema corporal (Función básica del cuerpo en el desarrollo psicomotor y educativo). Madrid: TEA.

Berruezo, P. P. (2000). Hacia un marco conceptual de la psicomotricidad a partir del desarrollo de su práctica en Europa y en España. Revista Interuniversitaria de Formación del Profesorado, 37, 21-33.

Berruezo, P. P. (2002). Las conductas motrices. En: Llorca, M. y otros: La Práctica Psicomotriz: una propuesta educativa mediante el cuerpo y el movimiento. Málaga: Aljibe.

Camps, C. (2008). La observación de la intervención del psicomotricista: actitudes y manifestaciones de la transferencia. Revista Interuniversitaria de Formación de Profesionales, 62, 123-154.

Camps, C. (2009). La especificidad de la Psicomotricidad: un arte para la estructuración de la persona. Revista Iberoamericana de Psicomotricidad y Técnicas Corporales, 33, 5-20.

Camps, C., Mila, J., García, L., Pecelli, M., Tomás, I., y Bottini, P. (2011). El psicomotricista en su cuerpo: de lo sensoriomotor a la transformación psíquica. Buenos Aires: Editorial Miño y Dávila.

Camps, C. (2012). Piel, envoltura, tocar y ser tocado. Revista Iberoamericana de Psicomotricidad y técnicas corporales, 37, 4-43.

Chaiklin, S. (2003). The zone of proximal development in Vygotsky's analysis of learning and instruction. Vygotsky's educational theory in cultural context, 1, 39-64. https://doi.org/10.1017/CBO9780511840975.004

Cró, M. L., Andreucci, L., Pereira, A., y Pinho, A. M. (2011). Psychomotricity, Health and Well-being in Childhood Education. En International Conference on Education and New Learning Technologies (EDULEARN) (pp.4716-4722).

Decreto 181/2008. Diari Oficial de la Generalitat de Catalunya. Barcelona, 09 de septiembre de 2008.

Del Arco, G. (2017). Práctica Psicomotriz Aucouturier en Educación Infantil. Desarrollo y aprendizaje a través del cuerpo en movimiento (Trabajo Final de Grado). Universidad Internacional de La Rioja. La Rioja.

D'Mello, S., Dale, R., y Graesser, A. (2012). Disequilibrium in the mind, disharmony in the body. Cognition \& Emotion, 26(2), 362-374. https://doi.org/10.1080/02699931.2011.575767

European Forum of Psychomotricity (2014). Declaración de París. Recuperado de https://aufop.blogspot.com/2014/07/paris-julio-de-2014-declaraciondel.html 
García, L., y Camps, C. (2006). Formación en la práctica: la adquisición de competencias profesionales en psicomotricidad. Revista Iberoamericana de Psicomotricidad y Técnicas Corporales, 24, 79-92.

Gutiérrez, L., Fontenla, E., y Cons, M. (2017). Mejora de la autoestima e inteligencia emocional a través de la psicomotricidad y de talleres de habilidades sociales. Sportis, Revista Técnico-Científica del Deporte Escolar, Educación Física y Psicomotricidad, 3(1), 187-205. https://doi.org/10.17979/sportis.2017.3.1.1813

Kambas, A., Fatouros, Y., Christoforidis, C., Venetsanou, F., Papageorgiou, P., Giannakidou, D., y Aggeloussis, N. (2010). The effects of Psychomotor Intervention, on Visual-Motor Control as Graphomotor aspect in preschool age. European Psychomotricity Journal, 3(1), 54-61.

Koppitz, E. (1984). El dibujo de la Figura Humana en los niños. Buenos Aires: Editorial Guadalupe.

Lapierre, A., Llorca, M., y Sánchez, J. (2015). Fundamentos de intervención en Psicomotricidad Relacional. Málaga: Ed. Aljibe.

Lelard, T., Stins, J., y Mouras, H. (2019). Postural responses to emotional visual stimuli. Neurophysiologie https://doi.org/10.1016/j.neucli.2019.01.005

Clinique.

Lupu, E. (2011). The role of motric activities in the psyco-motric development of preeschol children - future pupils. Procedia Social and Behavioral Sciences. 12, 457-464. https://doi.org/10.1016/j.sbspro.2011.02.056

Mamani, J. S. (2016). Nivel de psicomotricidad en niños de cuatro años de la institución educativa inicial 314 del distrito de Juliaca, provincia de San Román, región Puno, año 2016. (Tesis). Universidad Católica Los Ángeles Chimbote, Perú.

Mas, M. T. y Castellà, J. (2016). Can Psychomotricity improve cognitive abilities in infants?. Aloma, Revista de Psicologia, Ciències de l'Educació I de l'Esport. 34(1), 65-70.

Mayolas, M. y Reverter, J. (2015). Influencia de la edad y el género en los fenotipos y coeficientes de lateralidad en niños de 6 a 15 años. Apunts. Educación Física y Deportes, 120(2), 11-18. https://doi.org/10.5672/apunts.2014-0983.es.(2015/2).120.02

McCarthy, D. (1977). Escalas McCarthy de aptitudes y psicomotricidad para niños (MSCA). Madrid: TEA.

Mendiara, J. y Gil, P. (2003). La psicomotricidad. Evolución, corrientes y tendencias actuales. Sevilla: Wanceulen Editorial Deportiva, S.L.

Mila, J. (2005). La interdisciplina y los contenidos de la formación del psicomotricista. Revista Iberoamericana de Psicomotricidad y Técnicas Corporales. 19, 7-19.

Mila, J. (2008). De profesión psicomotricista. Madrid: Ed. Miño y Dávila editores.

Moreira, M. S., Almeida, G. N., y Marinho, S. M. (2016). Efectos de un programa de Psicomotricidad Educativa en niños en edad preescolar. Sportis, Revista Técnico-Científica del Deporte Escolar, Educación $\begin{array}{llll}\text { Física } y & \text { Psicomotricidad, 326-342. }\end{array}$ https://doi.org/10.17979/sportis.2016.2.3.1563

Newborg, J., Stock, J. R., y Wnek, L. (1998). Inventario de Desarrollo Battelle. Madrid: TEA. 
Picq. L. y Vayer. P. (1977). Educación psicomotriz y retraso mental. Barcelona: Editorial Científico Médica

Polacco, M. (2015). Psicoterapia quasi senza parole. Le relazioni di aiuto e le neuroscienze. Roma: Armando

Rivas, J. M. y Madrona, P. G. (2016). Psicomotricidad educativa. Wanceulen Editorial Deportiva, S.L.

Rota, J. (2015). La intervención psicomotriz: de la práctica al concepto. Barcelona: Ed. Octaedro.

Sánchez, J. y Llorca, M. (2008a). El rol del psicomotricista. Revista Interuniversitaria de Formación del Profesorado, 22, 35-60.

Sánchez, J. y Llorca, M. (2008b). Recursos y estrategias en psicomotricidad. Málaga: Ed. Aljibe.

Sassano, M. (2014). Cuerpo, función tónica y movimiento en Psicomotricidad. Buenos Aires: Miño y Dávila.

Sassano, M. (2015). El cuerpo como origen del tiempo y del espacio. Enfoques desde la Psicomotricidad. Buenos Aires: Miño y Dávila

Serrabona, J. (2016). Abordaje psicomotriz de las dificultades de desarrollo. Barcelona: Horsori.

Silva, M., Neves, G., y Moreira, S. (2016). Efectos de un programa de Psicomotricidad Educativa en niños en edad preescolar. Sportis, Revista Técnico-Científica del Deporte Escolar, Educación Física $y$ Psicomotricidad, 2(3), 326-342. https://doi.org/10.17979/sportis.2016.2.3.1563

Solís, A., Prieto, J. J., Nistal, P., y Vázquez, M. (2017). Percepción y aplicación de la psicomotricidad por parte del profesorado de la etapa infantil. Sportis, Revista Técnico-Científica del Deporte Escolar, Educación $\begin{array}{llll}\text { Física } \quad y \quad \text { Psicomotricidad, } & 3(1),\end{array}$ https://doi.org/10.17979/sportis.2017.3.1.1794

Terry, J. (2014). Análisis de la influencia de la metodología de la intervención psicomotriz sobre el desarrollo de las habilidades motrices en niños de 3 a 4 años (Tesis doctoral). Universidad de Murcia. Murcia.

Número de citas totales / Total references: 45 (100\%)

Número de citas propias de la revista / Journal's own references: $0(0 \%)$

Rev.int.med.cienc.act.fís.deporte - vol. 21 - número 81 - ISSN: 1577-0354 